Homology, Homotopy and Applications, vol. 9(2), 2007, pp.1-17

\title{
HOMOTOPY SPECTRAL SEQUENCES OF POINTED SIMPLICIAL SPACES
}

\author{
MICHAEL PALUCH
}

(communicated by J. F. Jardine)

\begin{abstract}
Using simplicial machinery, which is analogous to that developed by Bousfield-Kan, we describe a first quadrant homotopy spectral sequence for a termwise connected pointed simplicial space $X$ in terms of differential relations on the normalized homotopy of $X$ and establish the existence of a smash product pairing.
\end{abstract}

\section{Introduction}

As stated by Bousfield-Friedlander [2, Appendix B] "many constructions in algebraic topology can be achieved by first forming an appropriate bisimplicial set and then applying the diagonal functor." For example Goerss-Jardine [8, IV.5.1] use this technique to formulate the homology Serre spectral sequence. Following this approach one readily deduces that the Eilenberg-Zilber chain map $\nabla$ [13, page 234; 15, page 64] induces a pairing on the homology Serre spectral sequence; moreover, this pairing abuts to the homology cross product pairing (see [13, Ch.9.4; 15, XIII.8]).

Let $X$ be a pointed simplicial space. For simplicity we assume that $X$ is termwise connected; i.e., $X_{n}$ is a connected space for each $n \geqslant 0$. By [2, Theorem B.5] there is a first quadrant spectral sequence with $E_{s, t}^{2}(X) \cong \pi_{s}\left(\pi_{t} X\right)$ converging strongly to $\pi_{s+t} \operatorname{diag} X$. Using the basic properties of $\nabla$ (see [9, page 133], where it is called the Eilenberg-Mac Lane map) one readily deduces that $\nabla$ induces a pairing $E_{p, q}^{2}(X) \otimes$ $E_{s, t}^{2}(Y) \rightarrow E_{p+s, q+t}^{2}(X \wedge Y)$. Here we also assume that $Y$ is a termwise connected pointed simplicial space. Our key result (Theorem 4.6), which relies heavily on the seminal work of Bousfield-Kan [3], asserts that there is an induced spectral sequence pairing. We deduce its existence by analyzing the homological properties of a family of pointed simplicial spaces which serve as universal examples of relations on the normalized homotopy of $X$. The idea of using these examples to demonstrate the existence of the homotopy spectral sequence smash pairing is due to Pete Bousfield.

In Section 2 we discuss some well-known results relating bisimplicial abelian groups and first quadrant double chain complexes, and we reformulate the spectral

Supported in part by FCT through program POCTI/FEDER.

Received January 24, 2007, revised May 8, 2007; published on July 10, 2007.

2000 Mathematics Subject Classification: Primary 55T05; Secondary 18G30, 18G55, 55P65.

Key words and phrases: Homotopy spectral sequence, smash product pairing.

Copyright (C) 2007, International Press. Permission to copy for private use granted. 
sequence of a first quadrant double chain complexes in terms of a family of differential relations. In Section 3 we construct the homotopy spectral sequence of $X$ and compare it to the the spectral sequence derived from the spiral exact sequence of Dwyer-Kan-Stover [5] and to the Bousfield-Friedlander spectral sequence [2]. Finally, in Section 4, we show that $\nabla$ induces a smash pairing of the homotopy spectral sequence.

Throughout this note we use the term "space" to mean "simplicial set," and we assume that the reader is familiar with model categories as defined by Quillen [10].

\section{Acknowledgements}

The author heartily thanks Pete Bousfield for his unwavering help with the following work.

\section{The homology spectral sequence of a simplicial space}

\subsection{Simplicial abelian groups and chain complexes}

Let $\mathbf{s} \mathbf{A b}$ denote the category of simplicial abelian groups, and let $\mathbf{S}$ (resp. $\mathbf{S}_{*}$ ) denote the category of spaces (resp. pointed spaces). For $A \in \mathbf{s} \mathbf{A b}$, let $N_{*} A$ denote the normalized complex with

$$
N_{n} A=A_{n} \cap \operatorname{ker} d_{1} \cap \cdots \cap \operatorname{ker} d_{n}
$$

and differential $\partial$ induced by the remaining face operator $d_{0}$. The complex $N_{*} A$ is naturally isomorphic to the complex $N_{*}^{\prime} A$ defined in positive degrees by

$$
N_{n}^{\prime} A=A_{n} / \operatorname{im} s_{0}+\cdots+\operatorname{im} s_{n-1}
$$

and in degree 0 by $N_{0}^{\prime} A=A_{0}$ with differential $\partial=\sum_{i=0}^{n}(-1)^{i} d_{i}: N_{n}^{\prime} A \rightarrow N_{n-1}^{\prime} A$.

Neglecting the binary operation of a simplicial abelian group induces the forgetful functor $\mathbf{s} \mathbf{A} \mathbf{b} \rightarrow \mathbf{S}_{*}$, and, as is well known (e.g. [8, page 153]), there is a natural isomorphism $H_{*}(N A) \cong \pi_{*} A$. The forgetful functor has a left adjoint $\tilde{\mathbb{Z}}$ which sends a pointed space $X$ to the simplicial abelian group generated by the simplices of $X$ with the base vertex of $X$ and its degeneracies put equal to zero. For a pointed space $X$, we can use $\tilde{\mathbb{Z}}$ to obtain the reduced homology $\tilde{H}_{*}(X)=H_{*}(N \tilde{\mathbb{Z}} X)$; moreover, the adjunction map $X \rightarrow \tilde{\mathbb{Z}} X$ induces the Hurewicz homomorphism $h: \pi_{*}(X) \rightarrow$ $\tilde{H}_{*}(X)$ (e.g. $[8$, III.3]).

\subsection{Bisimplicial abelian groups and double chain complexes}

Let $\mathbf{b i s} \mathbf{A b}$ denote the category of bisimplicial abelian groups. One can think of a bisimplicial abelian group $B$ as a collection of abelian groups $B_{m, n}$ for $m, n \geqslant 0$ together with horizontal and vertical simplicial operators $\left(\alpha^{h}, \alpha^{v}\right)^{*}: B_{m, n} \rightarrow B_{p, q}$ for $\left(\alpha^{h}, \alpha^{v}\right):([p],[q]) \rightarrow([m],[n]) \in \boldsymbol{\Delta} \times \boldsymbol{\Delta}$ such that each horizontal operator commutes with each vertical operator. Here $\boldsymbol{\Delta}$ is the skeletal subcategory of finite ordered sets and non-decreasing maps consisting of objects $[n]=\{0,1, \ldots, n\}$ for $n \geqslant 0$. For $B \in$ bis $\mathbf{A b}$, the double normalized complex $N_{*} N_{*} B$ is supported in the 
first quadrant and is given by

$$
N_{m} N_{n} B=B_{m, n} \cap \operatorname{ker} d_{1}^{h} \cap \cdots \cap \operatorname{ker} d_{m}^{h} \cap \operatorname{ker} d_{1}^{v} \cap \cdots \cap \operatorname{ker} d_{n}^{v}
$$

with horizontal and vertical commuting differentials $\partial^{h}, \partial^{v}$ induced by the remaining face operators $d_{0}^{h}, d_{0}^{v}$. Let tot $B$ denote the total normalized complex with $(\text { tot } B)_{n}=$ $\bigoplus_{i \geqslant 0} N_{i} N_{n-i} B$ and differential $\left.\partial_{\text {tot }}\right|_{N_{i} N_{n-i} B}=\partial^{h}+(-1)^{i} \partial^{v}$.

The complex tot $B$ has an increasing canonically bounded [14, page 132] filtration with $F_{m}(\text { tot } B)_{n}=\bigoplus_{i \geqslant 0}^{m} N_{i} N_{n-i} B$. Thus the associated first quadrant spectral sequence with

$$
\begin{gathered}
E_{s, t}^{1}(B)=H_{s+t}\left(N_{s} N_{*} B\right) \cong N_{s} H_{t}(B) \\
E_{s, t}^{2}(B) \cong H_{s}\left(N_{*} H_{t}(B)\right) \cong \pi_{s} H_{t}(B) \\
d_{r}: E_{s, t}^{r}(B) \rightarrow E_{s-r, t+r-1}^{r}(B),
\end{gathered}
$$

where $N_{s} H_{t}(B)=H_{t}\left(B_{s}\right) \cap \operatorname{ker} d_{1} \cap \cdots \cap \operatorname{ker} d_{s}$, converges strongly to the homology of tot $B$. By the Eilenberg-Zilber-Cartier theorem [4, 2.9] there is a natural isomorphism $H_{*}(\operatorname{tot} B) \cong H_{*}(N \operatorname{diag} B)$, where $\operatorname{diag} B$ is the diagonal simplicial abelian group given by $(\operatorname{diag} B)_{n}=B_{n, n}$.

\subsection{Differential relations}

As in $[3,3.1]$ we shall find it more convenient to work with an explicit formulation of the homology spectral sequence of a bisimplicial abelian group rather than the implicit construction obtained from the filtration (cf. [1, page 14]).

Let $B$ be a bisimplicial abelian group. For $m \geqslant r \geqslant 1$, let

$$
\delta_{r} \subseteq N_{m} H_{n}(B) \times N_{m-r} H_{n+r-1}(B)
$$

denote the relation defined by $(x, y) \in \delta_{r}$ if and only if there is a zig-zag of elements connecting $x$ and $y$, in the sense that there are elements $x_{i} \in N_{m-i} N_{n+i} B$ for $0 \leqslant$ $i<r$ such that $0=\partial^{v} x_{0}, 0=\partial^{h} x_{i}+(-1)^{m-i-1} \partial^{v} x_{i+1}$ for $0 \leqslant i<r-1, x=\left[x_{0}\right]$ and $y=\left[\partial^{h} x_{r-1}\right]$. We view $\delta_{r}$ as the graph of a multivalued function

$$
d_{r}: N_{m} H_{n}(B) \rightsquigarrow N_{m-r} H_{n+r-1}(B),
$$

and write $d_{r} x=y$ if $(x, y) \in \delta_{r}$. Further details regarding relations can be found in [15, Appendix B]. Working in this spirit we define, respectively, the domain, image, kernel and indeterminacy of $d_{r}$ by

$$
\begin{aligned}
\operatorname{dom} d_{r} & =\left\{x \in N_{m} H_{n}(B) \mid d_{r} x=y \text { for some } y \in N_{m-r} H_{n+r-1}(B)\right\}, \\
\operatorname{im} d_{r} & =\left\{y \in N_{m-r} H_{n+r-1}(B) \mid d_{r} x=y \text { for some } x \in N_{m} H_{n}(B)\right\}, \\
\operatorname{ker} d_{r} & =\left\{x \in N_{m} H_{n}(B) \mid d_{r} x=0\right\}, \\
\operatorname{ind} d_{r} & =\left\{y \in N_{m-r} H_{n+r-1}(B) \mid d_{r} 0=y\right\} .
\end{aligned}
$$

A straightforward calculation shows that these relations have the following properties:

(i) $d_{1}$ corresponds to the differential of $N_{*} H_{q}(B)$,

(ii) $d_{r}$ is natural in $B$, 
(iii) $d_{r}$ is additive, i.e., $\delta_{r}$ is a subgroup,

(iv) $\operatorname{dom} d_{r+1}=\operatorname{ker} d_{r}$,

(v) ind $d_{r+1}=\operatorname{im} d_{r}$ and

(vi) $\operatorname{im} d_{r} \subseteq \operatorname{ker} d_{r}$.

Moreover

$$
E_{s, t}^{r}(B)=\left(N_{s} H_{t} B \cap \operatorname{ker} d_{r-1}\right) /\left(N_{s} H_{t} B \cap \operatorname{im} d_{r-1}\right), \text { for } r>1,
$$

and the relation $d_{r}$ induces the differential $d_{r}: E_{s, t}^{r}(B) \rightarrow E_{s-r, t+r-1}^{r}(B)$ of the homology spectral sequence.

\subsection{Convergence relations}

The target of the homology spectral sequence carries an induced filtration defined by

$$
F_{n} H_{*}(N \operatorname{diag} B)=\operatorname{im}\left[H_{*}\left(F_{n} \operatorname{tot} B\right) \rightarrow H_{*}(N \operatorname{diag} B)\right] .
$$

Let $e_{n}: N_{n} H_{t}(B) \rightsquigarrow H_{n+t}(N \operatorname{diag} B)$ denote the relation obtained by putting $e_{n} x=y$ whenever there is a cycle $b=\left(b_{0}, \ldots, b_{n}\right) \in F_{n}(\operatorname{tot} B)_{n+t}$ such that $x=\left[b_{n}\right]$ and $y=[\nabla b]$, where $\nabla:(\operatorname{tot} B)_{*} \rightarrow N_{*} \operatorname{diag} B$ is the Eilenberg-Mac Lane shuffle map [4, page 217; 9, pages 133-134]. These relations have the following properties:

(i) $e_{n}$ is natural in $B$,

(ii) $e_{n}$ is additive,

(iii) $e_{0}: N_{0} H_{t}(B) \rightsquigarrow H_{n}(N \operatorname{diag} B)$ is the homomorphism induced by the canonical map $B_{0} \rightarrow \operatorname{diag} B$ and the natural isomorphism $N_{0} H_{t}(B)=H_{t}\left(B_{0}\right)$,

(iv) $\operatorname{im} e_{n}=F_{n} H_{n+t}(N \operatorname{diag} B)$,

(v) ind $e_{n}=\operatorname{im} e_{n-1}$,

(vi) dom $e_{n}$ contains only infinite cycles in the sense that $e_{n} x=y \Longrightarrow d_{r} x=0$ for all $r$,

(vii) $\operatorname{ker} e_{n}$ contains only infinite boundaries in the sense that $e_{n} x=0 \Longrightarrow \exists q \geqslant 1$ such that $x \in \operatorname{im} d_{r}$ for all $q \leqslant r \leqslant n$.

Using these properties we deduce that each relation $e_{n}$ induces an isomorphism

$$
E_{n, t}^{\infty}(B) \stackrel{e_{n}}{\longrightarrow} F_{n} H_{n+t}(N \operatorname{diag} B) / F_{n-1} H_{n+t}(N \operatorname{diag} B) .
$$

\subsection{The homology spectral sequence}

For a pointed simplicial space $X$, let $\tilde{\mathbb{Z}} X$ denote the bisimplicial abelian group generated by the simplices of $X$ with the base point and its degeneracies put equal to zero. Let $\left\{E^{r}(X ; \tilde{\mathbb{Z}})\right\}$ denote the first quadrant spectral sequence obtained from $\tilde{\mathbb{Z}} X$. We have

$$
\begin{gathered}
E_{s, t}^{1}(X ; \tilde{\mathbb{Z}})=H_{s+t}\left(N_{s} N_{*} \tilde{\mathbb{Z}} X\right) \cong N_{s} \tilde{H}_{t}(X) \\
E_{s, t}^{2}(X ; \tilde{\mathbb{Z}}) \cong H_{s}\left(N_{*} \tilde{H}_{t}(\tilde{\mathbb{Z}} X)\right) \cong \pi_{s} \tilde{H}_{t}(X) \\
d_{r}: E_{s, t}^{r}(X ; \tilde{\mathbb{Z}}) \rightarrow E_{s-r, t+r-1}^{r}(X ; \tilde{\mathbb{Z}}),
\end{gathered}
$$

converges strongly to the reduced homology of $\operatorname{diag} X$. 


\section{The homotopy spectral sequence}

For a pointed cosimplicial space, Bousfield-Kan [3] constructed a homotopy spectral sequence generalizing the spectral sequence of a horizontally filtered second quadrant double chain complex. Here we present a simplicial analog of their construction. Let $\mathbf{s} \mathbf{S}_{*}$ denote the category of pointed simplicial spaces.

\subsection{Partial matching spaces}

For a space $K$ and a pointed space $Y$ the external half-smash product $K \ltimes Y$ is the pointed simplicial space given by $(K \underset{\sim}{\ltimes} Y)_{n}=\vee_{K_{n}} Y$. The functor $K \underset{\sim}{\ltimes}-: \tilde{\mathbf{S}}_{*} \rightarrow \mathbf{s} \mathbf{S}_{*}$ has a right adjoint $M_{K}$ which sends $X$ to the equalizer

$$
M_{K} X \rightarrow \prod_{[n] \in \Delta} \operatorname{hom}\left(K_{n}, X_{n}\right) \rightrightarrows \prod_{[m] \rightarrow[n] \in \Delta} \operatorname{hom}\left(K_{n}, X_{m}\right) .
$$

Simple calculations show that $M_{\Delta[n]} X=X_{n}$ and $M_{\partial \Delta[n]} X=M_{n} X$ is the usual matching space defined by

$$
M_{n} X=\left\{\left(x_{0}, \ldots, x_{n}\right) \mid x_{i} \in X_{n-1} \text { and } d_{i} x_{j}=d_{j-1} x_{i} \text { for } 0 \leqslant i<j \leqslant n\right\} .
$$

Furthermore, the inclusion $\partial \Delta[n] \hookrightarrow \Delta[n]$ induces the map $d=\left(d_{0}, \ldots, d_{n}\right): X_{n} \rightarrow$ $M_{n} X$.

For a model category $\mathbf{C}$, Reedy [11] showed that the category $\mathbf{s} \mathbf{C}$ of simplicial objects over $\mathbf{C}$ carries the structure of a model category. In the Reedy model structure a map $f: X \rightarrow Y$ of pointed simplicial spaces is (i) a weak equivalence if the map $f_{n}$ is a weak equivalence of spaces for each $n \geqslant 0$, (ii) a cofibration if it is injective and (iii) a fibration if the induced map $d: X_{n} \rightarrow Y_{n} \prod_{M_{n} Y} M_{n} X$ is a fibration of spaces for each $n \geqslant 0$. If $X$ is a fibrant pointed simplicial space, then $X_{n} \in \mathbf{S}_{*}$ is fibrant for each $n \geqslant 0$.

Theorem 3.1 (Reedy). The category $\mathbf{s}_{*}$ is a proper simplicial model category, where the simplicial structure is defined termwise.

Although Reedy does not actually consider proper model categories, in the sense of Bousfield-Friedlander $[2,1.2]$, the proof that $\mathbf{s} \mathbf{S}_{*}$ is proper is not difficult, cf. [2, $3.5 ; 8$, page 219].

\subsection{The Reedy model structure and the external half-smash product}

For a pointed space $X$, the pointed simplicial space $\Delta[0] \ltimes X$ is "constant", i.e., $(\Delta[0] \stackrel{\sim}{\ltimes} X)_{n}=X$ for all $n \geqslant 0$. The map $d^{i}: \Delta[0] \rightarrow \Delta[1]$ for $i=0,1$ is a trivial cofibration, yet the induced map

$$
X=\Delta[0] \underset{\sim}{\ltimes} X \rightarrow \Delta[1] \underset{\sim}{\ltimes} X \in \mathbf{s} \mathbf{S}_{*}
$$

is not a trivial cofibration of pointed simplicial spaces. Thus the external half-smash product functor $-\underset{\sim}{\ltimes}-: \mathbf{S} \times \mathbf{S}_{*} \rightarrow \mathbf{s} \mathbf{S}_{*}$ does not necessarily carry trivial cofibrations of spaces and cofibrations of pointed spaces to trivial cofibrations. The following relates the external half-smash product functor and the model structures of $\mathbf{S} \times \mathbf{S}_{*}$ and $\mathbf{s} \mathbf{S}_{*}$. 
Homology, Homotopy and Applications, vol. 9(2), 2007

Lemma 3.2. For a cofibration (resp. trivial cofibration) $f: X \rightarrow Y \in \mathbf{S}_{*}$ and a cofibration $i: K \rightarrow L \in \mathbf{S}$, the induced map

$$
(K \underset{\sim}{\ltimes} Y) \sqcup_{K \underset{\sim}{\ltimes} X}(L \underset{\sim}{\ltimes} X) \rightarrow L \underset{\sim}{\ltimes} Y
$$

is a cofibration (resp. trivial cofibration) of pointed simplicial spaces.

Proof. For a cofibration (resp. trivial cofibration) $f: X \rightarrow Y \in \mathbf{S}_{*}$, the induced map $\Delta[0] \stackrel{\sim}{\ltimes} f: X \rightarrow Y \in \mathbf{s} \mathbf{S}_{*}$ is a cofibration (resp. trivial cofibration) of pointed simplicial spaces. The proof now follows from [12, Corollary 7.4].

For a model category $\mathbf{C}$ and objects $X, Y \in \mathbf{C}$, let $[X, Y]=\operatorname{Hom}_{\mathbf{H o} \mathbf{C}}(X, Y)$. If $X$ is cofibrant and $Y$ is fibrant, then $[X, Y]=\operatorname{Hom}_{\mathbf{C}}(X, Y) / \sim$, where $\sim$ is a suitable homotopy relation on maps.

\subsection{External smash products}

For pointed spaces $X, Y$ the external smash product $X \wedge Y$ is the pointed simplicial space given by $(X \wedge Y)_{n}=X_{n} \wedge Y$. Note that if $K \rightarrow L \in \mathbf{S}$ is a cofibration and $X=L / K$, then there is a cocartesian square

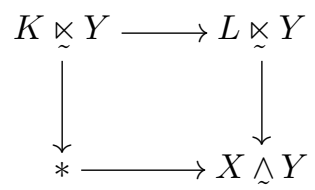

of pointed simplicial spaces in which the horizontal maps are cofibrations for each pointed space $Y$. The functor $X \wedge-$, like the external half-smash product functor $K \underset{\sim}{\ltimes}-$, has a right adjoint $C_{X}$ which sends a pointed simplicial space $Z$ to the end

$$
C_{X} Z \rightarrow \prod_{[n] \in \Delta} \operatorname{hom}_{*}\left(X_{n}, Z_{n}\right) \rightrightarrows \prod_{[m] \rightarrow[n] \in \Delta} \operatorname{hom}_{*}\left(X_{n}, Z_{m}\right),
$$

where $\operatorname{hom}_{*}(-,-): \mathbf{S}_{*} \times \mathbf{S}_{*} \rightarrow \mathbf{S}_{*}$ is the pointed mapping space functor and $X_{n}$ is the "discrete" pointed space of $n$-simplices of $X$. The functors $C_{X}$ and $M_{X}$ are related by the cartesian square of pointed spaces

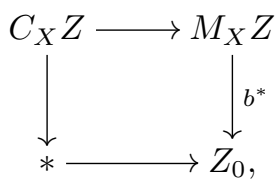

where $b: \Delta[0] \rightarrow X$ corresponds to the base point.

Let $K \rightarrow L \in \mathbf{S}_{*}$ be a cofibration. By Lemma 3.2 the induced map $M_{L} Z \rightarrow$ $M_{K} Z \in \mathbf{S}_{*}$ is a fibration for each fibrant pointed simplicial space $Z$, and as indicated 
by Goerss-Hopkins $[7,3.1 .1]$ there is a cartesian diagram

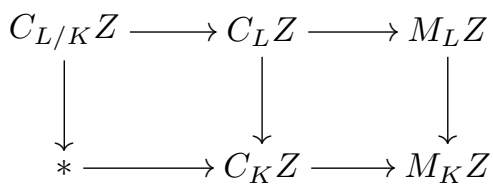

of pointed space in which the vertical maps are fibrations. Moreover, $K \wedge-$ preserves cofibrations and trivial cofibration. Thus $K \wedge \wedge_{-}: \mathbf{S}_{*} \leftrightarrows \mathbf{s ~}_{*}: C_{K}$ are Quillen adjoints, and for $Y$ a pointed fibrant simplicial space, there is a canonical isomorphism

$$
[K \stackrel{\sim}{\sim}, Y] \cong\left[X, C_{K} Y\right]
$$

\subsection{Universal examples for $d_{r}$ and $e_{n}$}

We now define pointed simplicial spaces $D_{m, n}^{r}$ and $S^{m, n}$ which will serve as universal examples for relations $d_{r}: N_{m} \pi_{n} X \rightsquigarrow N_{m-r} \pi_{n+r-1} X$ and $e_{n}: N_{n} \pi_{t} X \rightsquigarrow$ $\pi_{n+t} \operatorname{diag} X$ for a termwise connected pointed simplicial space $X$. For non-negative integers $m, n$, let $S^{m, n}=S^{m} \wedge S^{n}$, and let $D_{0, n}^{1}=S^{0, m}$. For $m \geqslant 1$ and $n \geqslant 0$, let $D_{m, n}^{1}$ denote the cofiber of

$$
V[m, 0]+\hat{\sim} S^{n} \rightarrow \Delta[m]_{+} \wedge S^{n} .
$$

Here $V[m, 0] \subseteq \partial \Delta[m]$ is the standard 0-horn spanned by the faces $d_{1} \iota_{m}, \ldots, d_{m} \iota_{m}$, and $S^{n}=\Delta[n] / \partial \Delta[n]$.

Using the Quillen adjoints $\Delta[m]_{+} \wedge-: \mathbf{S}_{*} \leftrightarrows \mathbf{s} \mathbf{S}_{*}:{ }_{n}$, where $-_{n}$ is the evaluation at $[n]$ functor, one readily proves

Proposition 3.3. For a fibrant pointed simplicial space $X$ and $n \geqslant 1$, there is a canonical isomorphism $N_{m} \pi_{n} X \cong\left[D_{m, n}^{1}, X\right]$. Moreover, the morphism

$$
\left[D_{m, n}^{1}, X\right] \rightarrow\left[D_{m-1, n}^{1}, X\right]
$$

induced by $d^{0}: \Delta[m-1] / V[m-1,0] \rightarrow \Delta[m] / V[m, 0]$, for $m>0$, or by $d^{0}: S^{0} \rightarrow$ $\Delta[1] / V[1,0]$, for $m=0$, corresponds to the differential

$$
\partial: N_{m} \pi_{n} X \rightarrow N_{m-1} \pi_{n} X
$$

of the normalized complex.

Let $j: D_{m-1, n}^{1} \rightarrow D_{m, n}^{1}$ denote the map induced by $d^{0}$. For $m>0, d^{0}$ factors as $\Delta[m-1] / V[m-1,0] \rightarrow S^{m-1} \rightarrow \Delta[m] / V[m, 0]$, and hence the map $j$ factors as

$$
D_{m-1, n}^{1} \rightarrow S^{m-1, n} \stackrel{j^{\prime}}{\longrightarrow} D_{m, n}^{1},
$$

with $j^{\prime}$ a cofibration. Clearly the cofiber of $j^{\prime}$ is $S^{m, n}$, and we may view $D_{m, n}^{1}$ as the extension of $S^{m, n}$ by $S^{m-1, n}$ that determines $\partial: N_{m} \pi_{n} X \rightarrow N_{m-1} \pi_{n} X$.

For $m \geqslant r \geqslant 1$ and $n \geqslant 0$, we use the familiar zig-zag of differentials of a first quadrant homology spectral sequence and define $D_{m, n}^{r} \in \mathbf{s} \mathbf{S}_{*}$ as the analogous 
extension of $S^{m, n}$ by $S^{m-r, n+r-1}$. In more precise terms $D_{m, n}^{r}$ is the pointed simplicial space with base vertex $*$ and non-degenerate simplices

$$
\left\{\iota_{s, t} \mid(s, t)=(m-i, n+i) \text { or }(m-i-1, n+i) \text { for } 0 \leqslant i<r\right\},
$$

where $\iota_{s, t} \in\left(D_{m, n}^{r}\right)_{s}$ is a $t$-simplex. Horizontal and vertical face maps are defined by

$$
d_{j}^{h} \iota_{m-i, n+i}=\left\{\begin{array}{ll}
* & \text { if } j>0 \\
\iota_{m-i-1, n+i} & \text { if } j=0
\end{array} \quad d^{v} \iota_{m-i, n+i}= \begin{cases}* & \text { if } j>0 \\
\iota_{m-i, n+i-1} & \text { if } j=0\end{cases}\right.
$$

There is a cofibration

$$
j^{\prime}: S^{m-r, n+r-1} \rightarrow D_{m, n}^{r}
$$

and a weak equivalence $D_{m, n}^{r} / S^{m-r, n+r-1} \rightarrow S^{m, n}$. Indeed, by the explicit description of the non-degenerate simplices of $D_{m, n}^{r}$, we see that $\left(D_{m, n}^{r}\right)_{s}$ is isomorphic to

$$
\left[\bigvee_{\sigma:[s] \rightarrow[m]} S^{n}\right] \vee\left[\bigvee_{\substack{\sigma:[s] \rightarrow[m-k] \\ 1 \leqslant k \leqslant r-1}} \frac{\Delta[n+k]}{V[n+k, 0]}\right] \vee\left[\bigvee_{\sigma:[s] \rightarrow[m-r]} S^{n+r-1}\right],
$$

where $\sigma:[s] \rightarrow[n]$ ranges over the surjective maps for each $s \geqslant n \geqslant 0$.

\subsection{Homotopy cofiberings of pointed simplicial spaces}

We say that

$$
A \stackrel{g}{\rightarrow} X \stackrel{h}{\rightarrow} Y
$$

is a homotopy cofibering in $\mathbf{s} \mathbf{S}_{*}$ if there is a commutative square

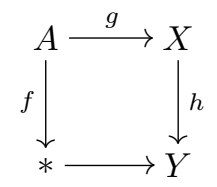

in $\mathbf{s S}_{*}$, and for some factorization $A \stackrel{i}{\rightarrow} B \stackrel{p}{\rightarrow} *$ of the canonical map $f$, with $i$ a cofibration and $p$ a weak equivalence, the canonical map $B \sqcup_{A} X \rightarrow Y$ is a weak equivalence. Since $\mathbf{s} \mathbf{S}_{*}$ is a proper model category [2, Appendix A], it is not hard to verify that if (4) is a homotopy cofibering and if $A \stackrel{j}{\rightarrow} \check{X} \stackrel{q}{\rightarrow} X$ is a factorization of $g$ with $j$ a cofibration and $q$ a weak equivalence, then the canonical map $\check{X} / A \rightarrow Y$ is a weak equivalence.

For $r>1$ the cofiber of the inclusion $i: D_{m, n}^{1} \rightarrow D_{m, n}^{r}$ is $D_{m-1, n+1}^{r-1}$, and we have a homotopy cofibering

$$
D_{m, n}^{1} \stackrel{i}{\rightarrow} D_{m, n}^{r} \rightarrow D_{m-1, n+1}^{r-1} .
$$

For $m>r \geqslant 1$, let $j: D_{m-r, n+r-1}^{1} \rightarrow D_{m, n}^{r} \in \mathbf{s} \mathbf{S}_{*}$ denote the composition of the projection $D_{m-r, n+r-1}^{1} \rightarrow S^{m-r, n+r-1}$ and (2). 
Lemma 3.4. There is a commutative diagram in $\mathbf{s} \mathbf{S}_{*}$ in which the diagonal maps are homotopy cofibering

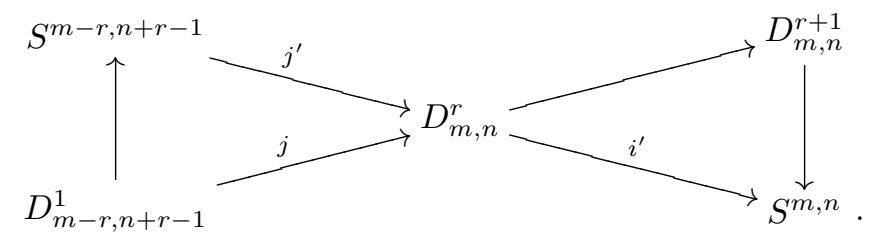

Proof. For $r \geqslant 1$ the cofacial operator $d^{0}:[n+r-1] \rightarrow[n+r]$ induces a pointed cofibration $S^{n+r-1} \hookrightarrow \Delta[n+r] / V[n+r, 0]$, and for $m>r$ we obtain a push-out square

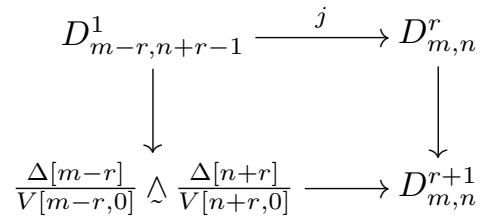

in which the vertical maps are pointed cofibrations. The proof of the lemma follows by a simple calculation and the observation that

$$
(\Delta[m-r] / V[m-r, 0]) \wedge(\Delta[n+r] / V[n+r, 0]) \rightarrow *
$$

is a weak equivalence.

\subsection{Differential relations in terms of $D_{m, n}^{l}$}

Let $\gamma: \mathbf{s} \mathbf{S}_{*} \rightarrow \mathbf{H o s} \mathbf{S}_{*}$ be a localization functor which is the identity on objects. For a pointed termwise connected simplicial space $X$, we use Proposition 3.3 to identify $N_{m} \pi_{n} X$ and $\left[D_{m, n}^{1}, X\right]$, and we define the pointed relation

$$
d_{l}: N_{m} \pi_{n} X \rightsquigarrow N_{m-l} \pi_{n+l-1} X, \text { for } m \geqslant l \geqslant 1,
$$

by $d_{l} x=y$ if and only if there is a map $f: D_{m, n}^{l} \rightarrow X \in \mathbf{H o s}_{*}$ such that $f \circ \gamma(i)=$ $x$ and $f \circ \gamma(j)=y$.

In general a weak equivalence $S^{n+t} \rightarrow S^{n} \wedge S^{t} \in \mathbf{S}_{*}$ does not exist; nevertheless, using the geometric realization functor $|-|$ and its right adjoint, the singular complex functor sing $[9, \S 14]$, as well as the standard homeomorphism [15, page 107] $\left|S^{n}\right| \wedge\left|S^{t}\right| \cong\left|S^{n+t}\right|$, one can construct a canonical zig-zag of weak equivalences $S^{n+t} \rightarrow \cdots \leftarrow S^{n} \wedge S^{t} \in \mathbf{S}_{*}$. Thus we may identify $\left[S^{n} \wedge S^{t}, \operatorname{diag} X\right]$ and $\pi_{n+t} \operatorname{diag} X$. Since $\operatorname{diag}: \mathbf{s} \mathbf{S}_{*} \rightarrow \mathbf{S}_{*}$ carries cofibrations and weak equivalences to cofibrations and weak equivalences respectively, a total left derived functor $\mathcal{L}$ diag exists; moreover, we may assume that $\mathcal{L} \operatorname{diag} X=\operatorname{diag} X$ for each $X \in \mathbf{s} \mathbf{S}_{*}$.

For a termwise connected pointed simplicial space $X$, we define

$$
e_{n}: N_{n} \pi_{t} X \rightsquigarrow \pi_{n+t} \operatorname{diag} X \text {, for } 0 \leqslant n \text { and } 1 \leqslant t,
$$

by $e_{n} x=y$ if and only if there is a map $f: S^{n, t} \rightarrow X \in \mathbf{H o s} \mathbf{S}_{*}$ such that

$$
x=f \circ \gamma\left(i^{\prime}\right) \in\left[D_{n, t}^{1}, X\right] \text { and } y=\mathcal{L} \operatorname{diag}(f) .
$$


Theorem 3.5. For a termwise connected pointed simplicial space $X$, the relations $d_{l}$ and $e_{n}$ are the unique relations which coincide with the relations of Sections 2.3 and 2.4 whenever $X$ is the underlying pointed simplicial space of a bisimplicial abelian group and which have the following properties:

(i) $d_{1}: N_{m} \pi_{m} X \rightsquigarrow N_{m-1} \pi_{n} X$ is the differential of the normalized chain complex $N_{*} \pi_{n} X$ and $e_{0}: N_{0} \pi_{t} X \rightsquigarrow \pi_{t} \operatorname{diag} X$ is the homomorphism induced by the canonical map $X_{0} \rightarrow \operatorname{diag} X$,

(ii) $d_{l}$ and $e_{n}$ are natural in $X$,

(iii) $d_{l}$ and $e_{n}$ are additive,

(iv) $\operatorname{im} e_{n} \subseteq \operatorname{im} e_{n+1}$,

(v) $\operatorname{dom} d_{l+1}=\operatorname{ker} d_{l}$ and ind $e_{n}=\operatorname{im} e_{n-1}$,

(vi) ind $d_{l+1}=\operatorname{im} d_{l}$ and $\operatorname{ker} e_{n}$ is the image of the differential relation

$$
d_{t}: N_{n+t} \pi_{1} X \rightarrow N_{n} \pi_{t} X
$$

(vii) $\operatorname{dom} e_{n}$ contains only infinite cycles.

Proof. Property (ii) is obvious. For $d_{1}$, property (i) follows by Proposition 3.3. Using the natural isomorphisms $S^{0} \wedge S^{t}=S^{t}, D_{0, t}^{1}=\Delta[0] \underset{\sim}{\ltimes} S^{t}$ and $\left[S^{0} \wedge S^{t}, X\right]=$ $\left[S^{q}, X_{0}\right]=N_{0} \pi_{q} X$, we obtain (i) for $e_{0}$. For $n \geqslant 1$,

$$
D_{m, n}^{1} \stackrel{\gamma(i)}{\longrightarrow} D_{m, n}^{l} \stackrel{\gamma(j)}{\longleftarrow} D_{m-l, n+l-1}^{1} \in \mathbf{H o s} \mathbf{S}_{*}
$$

are maps of co-groups and $\mathcal{L} \operatorname{diag}:\left[S^{n, t}, X\right] \rightarrow \pi_{n+t} \operatorname{diag} X$ is additive. Property (iii) follows for $d_{l}$ by the general theory of simplicial groups (e.g. [9, §17]). By the work of Dwyer-Kan-Stover [5, 5.5 and 5.8], we deduce that there is an exact sequence

$$
\left[D_{n+t, 1}^{1}, X\right] \stackrel{\left(j^{\prime}\right)^{*}}{\longrightarrow}\left[S^{n+t-1,1}, X\right] \stackrel{\mathcal{L} \operatorname{diag}}{\longrightarrow} \pi_{n+t} \operatorname{diag} X \rightarrow 0
$$

for each $n+t \geqslant 1$, and (iii) readily follows for $e_{n}$. By the proof of $[5,6.3]$, we see that that $\mathcal{L} \operatorname{diag}:\left[S^{n, t}, X\right] \rightarrow \pi_{n+t} \operatorname{diag} X$ factors as

$$
\left[S^{n, t}, X\right] \stackrel{k^{*}}{\longrightarrow}\left[S^{n+1, t-1}, X\right] \stackrel{k^{*}}{\longrightarrow} \cdots \stackrel{k^{*}}{\longrightarrow}\left[S^{n+t-1,1}, X\right] \rightarrow \pi_{n+t} \operatorname{diag} X,
$$

where $k: S^{k, l} \rightarrow S^{k-1, l+1}$ is obtained from the homotopy cofibering

$$
S^{k-1, l} \stackrel{j^{\prime}}{\rightarrow} D_{k, l}^{1} \stackrel{i^{\prime}}{\rightarrow} S^{k, l} .
$$

This proves property (iv). Using (5), Lemma 3.4, (6), as well as the homotopy cofibration sequence

$$
D_{n, t}^{1} \stackrel{\gamma\left(i^{\prime}\right)}{\longrightarrow} S^{n, t} \rightarrow \Sigma D_{n-1, t}^{1} \in \mathbf{H o s} \mathbf{S}_{*},
$$

we deduce properties (v) and (vi). To prove property (vii) we factor the map $i^{\prime}: D_{n, t}^{1} \rightarrow S^{n, t}$ as

$$
D_{n, t}^{1} \rightarrow D_{n, t}^{2} \rightarrow \cdots \rightarrow D_{n, t}^{n} \rightarrow S^{n, t}
$$

To prove these relations coincide with those of Sections 2.3 and 2.4, we use the fact, which follows from Reedy [11], that the category bis Ab has the structure of 
a model category in which a map $f: A \rightarrow B$ is a weak equivalence (resp. fibration) whenever the underlying map $f \in \mathbf{s} \mathbf{S}_{*}$ is a weak equivalence (resp. fibration). In particular the total derived functors $\mathcal{L} \tilde{\mathbb{Z}}:$ Ho s $\mathbf{S}_{*} \leftrightarrows \mathbf{H o}$ bis $\mathbf{A b}: \mathcal{R}$ exist and form an adjoint pair. Here $\mathcal{R}$ is a total right derived functor of the forgetful functor. Since each bisimplicial abelian group is fibrant and each simplicial space is cofibrant, there are natural isomorphisms

$$
\begin{aligned}
{\left[\tilde{\mathbb{Z}} D_{m, n}^{l}, B\right]_{\text {Ho bis Ab }} } & \cong\left[D_{m, n}^{l}, B\right]_{\text {Hos }} \mathbf{S}_{*} \\
{\left[\tilde{\mathbb{Z}} S^{n, t}, B\right]_{\text {Ho bis Ab }} } & \cong\left[S^{n, t}, B\right]_{\text {Hos }} \mathbf{S}_{*}
\end{aligned}
$$

for each $B \in \mathbf{b i s} \mathbf{A b}$. Using the explicit description of the non-degenerate simplices of $D_{m, n}^{l}$ and the identity $S^{n, t}=S^{n} \wedge S^{t}$, we see that respective the homotopy differentials of Section 3.6 agree with those of Sections 2.3 and 2.4. By a straightforward calculation using the pointed simplicial spaces $D_{m, n}^{l}$ and $S^{n, t}$ we deduce uniqueness.

\subsection{The homotopy spectral sequence}

We define the homotopy spectral sequence of a termwise connected pointed simplicial space $X$ to be the first quadrant spectral sequence $\left\{E^{r}(X)\right\}$ with

$$
\begin{aligned}
& E_{s, t}^{1}(X)=N_{s} \pi_{t} X \\
& E_{s, t}^{r}(X)=N_{s} \pi_{t} X \cap \operatorname{ker} d_{r-1} / N_{s} \pi_{t} X \cap \operatorname{im} d_{r-1} \quad \text { for } r>1 .
\end{aligned}
$$

The differential $d_{r}: E_{s, t}^{r}(X) \rightarrow E_{s-r, t+r-1}^{r}(X)$ is the homomorphism induced by the differential of Section 3.6. For $r>s$ we have a surjective homomorphism $E_{s, t}^{r}(X) \rightarrow$ $E_{s, t}^{r+1}(X)$. Thus the $E^{\infty}$-term is given by $E_{s, t}^{\infty}(X)=\operatorname{colim}_{r>s} E_{s, t}^{r}(X)$. Let

$$
F_{n} \pi_{*} \operatorname{diag} X=\operatorname{im} e_{n} \cap \pi_{*} \operatorname{diag} X .
$$

By Theorem 3.5, $F$ is an increasing filtration of $\pi_{t} \operatorname{diag} X$ and $e_{n}$ induces an isomorphism

$$
E_{n, t}^{\infty}(X) \stackrel{\sim}{\longrightarrow} F_{n} \pi_{n+t} \operatorname{diag} X / F_{n-1} \pi_{n+t} \operatorname{diag} X .
$$

Thus the homotopy spectral sequence $\left\{E^{r}(X)\right\}$ converges strongly to $\pi_{*} \operatorname{diag} X$.

Corollary 3.6. For a pointed termwise connected simplicial space $X$, the adjunction map $X \rightarrow \tilde{\mathbb{Z}} X$ induces a map hrom the homotopy spectral sequences $\left\{E^{r}(X)\right\}$ to the reduced homology spectral sequence $\left\{E^{r}(X, \tilde{\mathbb{Z}})\right\}$. At the $E^{1}$-level $h: N_{m} \pi_{n} X \rightarrow$ $N_{m} \tilde{H}_{n}(X)$ is induced by the Hurewicz homomorphism and

$$
h:\left\{E^{r}(X)\right\} \rightarrow\left\{E^{r}(X, \tilde{\mathbb{Z}})\right\}
$$

abuts to the Hurewicz homomorphism $h: \pi_{t} \operatorname{diag} X \rightarrow \tilde{H}_{t}(\operatorname{diag} X)$. In particular, the Hurewicz homomorphism is a morphism of filtered groups.

Let $X$ be a termwise connected pointed fibrant simplicial space. Dwyer-KanStover $[5,8.2]$ derive a homotopy exact couple and a spectral sequence with

$$
E_{s, t}^{1}(X)=\pi_{t} C_{S^{s}} X \cong N_{s} \pi_{t} X
$$

from the family of fibrations $C_{\Delta[n+1] / V[n+1,0]} X \rightarrow C_{S^{n}} X$, induced by the cofibra- 
tion $d^{0}: S^{n} \rightarrow \Delta[n+1] / V[n+1,0]$ for $n \geqslant 0$, and show that exact couple extends naturally to all termwise connected pointed simplicial spaces.

Proposition 3.7. For a termwise connected pointed simplicial space $X$, the homotopy spectral sequence of Section 3.7 and the Dwyer-Kan-Stover spectral sequence are naturally isomorphic.

Proof. For a termwise connected pointed fibrant simplicial space $X$, the DwyerKan-Stover spectral sequence is derived from the fibration sequence

$$
C_{\Delta[m+1] / \partial \Delta[m+1]} X \rightarrow C_{\Delta[m+1] / \partial V[m+1,0]} X \rightarrow C_{\Delta[m] / \partial \Delta[m]} X .
$$

Using (1) we deduce that this spectral sequence can be derived from the exact couple

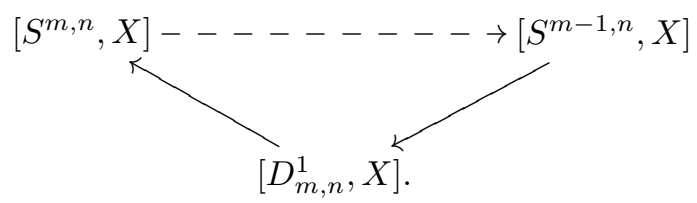

It follows that the associated differential relations are the same as those of Section 3.6. Thus the two spectral sequences are naturally isomorphic.

For a termwise connected fibrant pointed simplicial space $X$, let $\left(P_{t} X\right)_{n} \in \mathbf{S}_{*}$ denote the $t^{\text {th }}$-Postinkov sections of $X_{n}$ and let $\left(F_{t} X\right)_{n}$ denote the fiber of the canonical map $\left(P_{t} X\right)_{n} \rightarrow\left(P_{t-1} X\right)_{n}$. By [2, B4] there is a homotopy fibration $\operatorname{diag} F_{t} X \rightarrow$ $\operatorname{diag} P_{t} X \rightarrow \operatorname{diag} P_{t-1} X$. Thus there is an exact couple

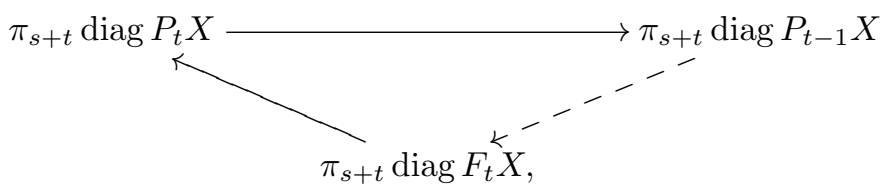

with $\pi_{s+t} \operatorname{diag} F_{t} X \cong \pi_{s}\left(\pi_{t} X\right)$ [2, B5]. The corresponding spectral sequence is a special case of the Bousfield-Friedlander spectral sequence. By [5, 8.4] the DwyerKan-Stover spectral sequence is naturally isomorphic to the Bousfield-Friedlander spectral sequence.

Corollary 3.8. For a termwise connected simplicial space, the homotopy spectral sequence of Section 3.7 is naturally isomorphic to the Bousfield-Friedlander spectral sequence.

\section{Pairings}

\subsection{The shuffle map $\nabla$}

For $m, p \geqslant 0$ with $m+p>0$ an ( $m, p)$-shuffle is a permutation $\sigma$ of $\{0, \ldots, m+p-1\}$ such that $\sigma(i)<\sigma(j)$ for $0 \leqslant i<j \leqslant m-1$ and for $m \leqslant i<j \leqslant m+p-1$. Let $\Sigma(m, p)$ denote the collection of $(m, p)$-shuffles. The 
signature or sign of $\sigma \in \Sigma(m, p)$ is the sign of the permutation $\sigma$; it is given by $\operatorname{sgn}(\sigma)=(-1)^{\sum_{i=0}^{m-1} \sigma(i)-i}$.

For simplicial abelian groups $A$ and $B$, the collection of shuffle maps induces an injective quasi-isomorphism $\left[4,2.9\right.$ and 2.15; 9, page 134] $\nabla: N_{*} A \otimes N_{*} B \rightarrow$ $N_{*}(A \otimes B)$ which sends $a \in N_{m} A, b \in N_{p} B$ to

$$
\sum_{\sigma \in \Sigma(m, p)} \operatorname{sgn}(\sigma) s_{\sigma(m+p-1)} \cdots s_{\sigma(m)} a \otimes s_{\sigma(m-1)} \cdots s_{\sigma(0)} b .
$$

The map $\nabla$ is graded commutative in the sense that

$$
\tau_{*} \nabla(a \otimes b)=(-1)^{m p} \nabla(b \otimes a),
$$

where $\tau: A \otimes B \rightarrow B \otimes A$ the twist map and $a \in N_{m} A, b \in N_{p} B$. Moreover, for maps of simplicial abelian groups $f: A \rightarrow A^{\prime}$ and $g: B \rightarrow B^{\prime}$ we clearly have

$$
(f \otimes g) \circ \nabla(a \otimes b)=\nabla(f(a) \otimes g(b)) .
$$

Thus $\nabla$ induces a natural graded commutative pairing

$$
H_{m}(A) \otimes H_{p}(B) \stackrel{\nabla}{\longrightarrow} H_{m+p}(A \otimes B) .
$$

Note that this pairing also is associative.

For bisimplicial abelian groups $A$ and $B$ the composition

$$
N_{m} H_{n} A \otimes N_{p} H_{q} B \stackrel{\nabla^{g}}{\longrightarrow} N_{m+p} H_{n}(A) \otimes N_{m+p} H_{q}(B) \stackrel{\nabla}{\longrightarrow} N_{m+p} H_{n+q}(A \otimes B),
$$

where $\nabla^{g}$ is the graded shuffle map given by

$$
\nabla^{g}(a \otimes b)=(-1)^{n p} \sum_{\sigma \in \Sigma(m, p)} \operatorname{sgn}(\sigma) s_{\sigma(m+p-1)} \cdots s_{\sigma(m)} a \otimes s_{\sigma(m-1)} \cdots s_{\sigma(0)} b,
$$

is an associative and graded commutative pairing.

Theorem 4.1. The pairing (7) induces a pairing of spectral sequences

$$
E_{m, n}^{r}(A) \otimes E_{p, q}^{r}(B) \stackrel{-\wedge-}{\longrightarrow} E_{m+p, n+q}^{r}(A \otimes B)
$$

with the following properties:

(i) the pairing on $E^{1}$ is the pairing (7),

(ii) the differential $d_{r}$ satisfies the Leibniz relation

$$
d_{r}(x \wedge y)=d_{r} x \wedge y+(-1)^{m+n} x \wedge d_{r} y
$$

for $x \in E_{m, n}^{r}(A)$,

(iii) the pairing on $E^{r+1}$ is induced by the pairing on $E^{r}$,

(iv) the pairing on $E^{\infty}$ is compatible with the filtration of Section 2.4,

(v) the pairing is associative and graded commutative with sign $(-1)^{(m+n)(p+q)}$ for $r \geqslant 1$.

Proof. The proof of the theorem follows readily from the observations that the pairing of two cycles is a cycle, the pairing of a boundary and a cycle is a boundary, and the shuffle maps carry $F_{m}(\operatorname{tot} A)_{t} \otimes F_{n}(\operatorname{tot} B)_{s}$ to $F_{m+n}(\operatorname{tot}(A \otimes B))_{s+t}$. 


\subsection{The induced pairing}

Using the natural isomorphism

$$
D_{m, n}^{1} \wedge D_{p, q}^{1} \cong\left(\frac{\Delta[m]}{V[m, 0]} \wedge \frac{\Delta[p]}{V[p, 0]}\right) \wedge\left(S^{n} \wedge S^{q}\right) \quad \text { for } m, n, p, q>0
$$

and the Hurewicz isomorphism theorem, we obtain an isomorphism

$$
\left[D_{m+p, n+q}^{1}, D_{m, n}^{1} \wedge D_{p, q}^{1}\right] \cong N_{m+p} \tilde{\mathbb{Z}}\left(\frac{\Delta[m] \times \Delta[p]}{V[m, 0] \times \Delta[p] \cup V[m, 0] \times \Delta[p]}\right) .
$$

Let

$$
D_{m+p, n+q}^{1} \stackrel{\nabla}{\longrightarrow} D_{m, n}^{1} \wedge D_{p, q}^{1} \in \mathbf{H o s} \mathbf{S}_{*}
$$

denote the element corresponding to the image of $(-1)^{n p}\left[\iota_{m}\right] \otimes\left[\iota_{p}\right]$ under the shuffle map

$$
N_{*} \tilde{\mathbb{Z}}\left(\frac{\Delta[m]}{V[m, 0]}\right) \otimes N_{*} \tilde{\mathbb{Z}}\left(\frac{\Delta[p]}{V[p, 0]}\right) \stackrel{\nabla}{\longrightarrow} N_{*} \tilde{\mathbb{Z}}\left(\frac{\Delta[m] \times \Delta[p]}{V[m, 0] \times \Delta[p] \cup V[m, 0] \times \Delta[p]}\right) .
$$

Let $X$ and $Y$ be fibrant pointed simplicial spaces. Using the natural isomorphism of Proposition 3.3 and (8) we obtain a natural map

$$
N_{m} \pi_{n} X \wedge N_{p} \pi_{q} Y \stackrel{\nabla^{*}}{\longrightarrow} N_{m+p} \pi_{n+q}(X \wedge Y) .
$$

Proposition 4.2. For fibrant pointed simplicial spaces $X, Y, \nabla^{*}$ is a bilinear associative graded commutative pairing of complexes in the sense that

$$
\begin{aligned}
\tau_{*} \nabla^{*}(x \wedge y) & =(-1)^{(m+n)(p+q)} \nabla^{*}(y \wedge x) \\
d_{1} \nabla^{*}(x \wedge y) & =\nabla^{*}\left(d_{1} x \wedge y\right)+(-1)^{m+n} \nabla^{*}\left(x \wedge d_{1} y\right),
\end{aligned}
$$

where $\tau: X \wedge Y \rightarrow Y \wedge X$ is the standard twist map and $x \in N_{m} \pi_{n} X, y \in N_{p} \pi_{q} Y$; moreover, the Hurewicz map carries this pairing to the pairing of (7).

Proof. Using the familiar properties of the relative form of the (graded) EilenbergZilber-Cartier map (cf. [13, pages 232-234]) one readily deduces that $\nabla^{*}$ is a bilinear, associative and graded commutative pairing of complexes. To prove the last assertion we use the fact that for each $(m, p)$-shuffle $\sigma$, the map

$$
s_{\sigma(m+p-1)} \cdots s_{\sigma(m)}: N_{m} \tilde{H}_{n}(X) \rightarrow N_{m+p} \tilde{H}_{n}(X),
$$

corresponds to

$$
D_{m+p, n}^{1} \stackrel{s^{\sigma(m)} \ldots s^{\sigma(m+p-1)} \stackrel{\sim}{\hookrightarrow}}{\longrightarrow} D_{m, n}^{1} \rightarrow \tilde{\mathbb{Z}} X \in \mathbf{H o s} \mathbf{S}_{*}
$$

under the canonical isomorphism $\left[D_{m, p}^{1}, \tilde{\mathbb{Z}} X\right] \cong N_{m} \tilde{H}_{q}(X)$.

By Proposition 4.2 we obtain an induced associative and graded commutative pairing

$$
E_{p, q}^{2}(X) \otimes E_{s, t}^{2}(Y) \rightarrow E_{p+s, q+t}^{2}(X \wedge Y)
$$

which is compatible with the homology pairing of Theorem 4.1.

In order to show that there is a pairing on the homotopy spectral sequence it suffices to analyze the homotopy spectral sequences of $D_{p, q}^{r} \wedge D_{s, t}^{r}$ in a limited region of the first quadrant. 
Lemma 4.3. For $m \geqslant 0$, the pointed spaces $\pi_{m}\left(S^{p, q} \wedge S^{s, t}\right)$ and $\pi_{m} S^{p+s, q+t}$ are weakly equivalent.

Proof. Let $\Gamma^{0}$ denote the skeletal subcategory of pointed sets and pointed maps consisting of objects $n^{+}=\{0,1, \ldots, n\}$, for $n \geqslant 0$, pointed by 0 . Consider the functor $F: \Gamma^{0} \rightarrow \mathbf{S}_{*}$ defined by $F\left(n^{+}\right)=\pi_{m}\left(\vee_{i=1}^{n} S^{q+t}\right)$. Here we view the pointed set $\pi_{m}\left(\vee_{i=1}^{n} S^{q+t}\right)$ as a "constant" pointed space. The functor $F$ prolongs to a functor $F: \mathbf{S}_{*} \rightarrow \mathbf{S}_{*}$ with $F(K)$ the pointed space whose set of $n$-simplices is $F(K)_{n}=$ $\pi_{m}\left(K_{n} \wedge S^{q+t}\right)$ for $K \in \mathbf{S}_{*}$. By [2, Proposition 4.9] each weak equivalence $f: K \rightarrow$ $L \in \mathbf{S}_{*}$ induces a weak equivalence $f_{*}: F(K) \rightarrow F(L)$. Since the pointed spaces $S^{p+s}$ and $S^{p} \wedge S^{s}$ have the same weak homotopy type, the proof of the lemma follows.

Consider the homotopy cofibering $S^{p-r, q+r-1} \stackrel{j^{\prime}}{\rightarrow} D_{p, q}^{r} \stackrel{i^{\prime}}{\rightarrow} S^{p, q}$ of Lemma 3.4.

Lemma 4.4. The map $\left(i^{\prime} \wedge i^{\prime}\right)_{*}: \pi_{n}\left(D_{p, q}^{r} \wedge D_{s, t}^{r}\right) \rightarrow \pi_{n}\left(S^{p, q} \wedge S^{s, t}\right)$ is an isomorphism for $0 \leqslant n \leqslant q+t+r-1$ and onto for $n=q+t+r$.

Proof. This follows from the Hilton-Milnor theorem [1, page 131] and (3).

Let $\operatorname{Cyl}\left(i^{\prime} \wedge i^{\prime}\right) \in \mathbf{s} \mathbf{S}_{*}$ denote the mapping cylinder of $i^{\prime} \wedge i^{\prime}$ constructed in the usual manner [15, page 23] using the termwise simplicial structure. Using (3) one readily deduces that there is a short exact sequence

$$
\pi_{n}\left(\operatorname{Cyl}\left(i^{\prime} \wedge i^{\prime}\right), D_{p, q}^{r} \wedge D_{s, t}^{r}\right) \longmapsto \pi_{n-1}\left(D_{p, q}^{r} \wedge D_{s, t}^{r}\right) \rightarrow \pi_{n-1}\left(S^{p, q} \wedge S^{s, t}\right)
$$

of simplicial abelian groups for each $n>2$.

\section{Proposition 4.5.}

$$
E_{m, n}^{2}\left(D_{p, q}^{r} \wedge D_{s, t}^{r}\right) \cong \begin{cases}\mathbb{Z} & \text { if } m=p+s \text { and } n=q+t, \\
0 & \text { if }\left\{\begin{array}{l}
p+s-r<m<p+s \text { and } \\
q+t<n<q+t+r-1,
\end{array}\right. \\
\mathbb{Z} \oplus \mathbb{Z} & \text { if } m=p+s-r \text { and } n=q+t+r-1 .\end{cases}
$$

Proof. By Lemmas 4.3 and 4.4 we see that

$$
E_{m, n}^{2}\left(D_{p, q}^{r} \wedge D_{s, t}^{r}\right) \cong \tilde{H}_{m}\left(S^{p+s}, \pi_{n} S^{q+t}\right) \text { for } n<q+t+r-1 .
$$

Using the relative Hurewicz isomorphism theorem we obtain an isomorphism

$$
\pi_{q+t+r}\left(\operatorname{Cyl}\left(i^{\prime} \wedge i^{\prime}\right), D_{p, q}^{r} \wedge D_{s, t}^{r}\right) \cong H_{q+r+t}\left(\operatorname{Cyl}\left(i^{\prime} \wedge i^{\prime}\right), D_{p, q}^{r} \wedge D_{s, t}^{r}\right) .
$$

By the long exact homology sequence of $\left.\left(\operatorname{Cyl}\left(i^{\prime} \wedge i^{\prime}\right), D_{p, q}^{r} \wedge D_{s, t}^{r}\right)\right)$ and the weak equivalence $\operatorname{Cyl}\left(i^{\prime} \wedge i^{\prime}\right) \simeq S^{p, q} \wedge S^{s, t}$ we get $E_{p+s-r, q+t+r-1}^{2}\left(D_{p, q}^{r} \wedge D_{s, t}^{r}\right) \cong \mathbb{Z} \oplus \mathbb{Z}$.

Theorem 4.6. For termwise connected pointed simplicial spaces $X$ and $Y$, there is a natural pairing of spectral sequences

$$
E_{p, q}^{r}(X) \otimes E_{s, t}^{r}(Y) \rightarrow E^{r}(X \wedge Y), \quad r \geqslant 2
$$

such that 
(i) $d_{r}(x \wedge y)=\left(d_{r} x \wedge y\right)+(-1)^{p+q}\left(x \wedge d_{r} y\right)$,

(ii) the pairing on $E^{2}$ is the pairing (9),

(iii) the pairing on $E^{r}$ induces the pairing on $E^{r+1}$,

(iv) the pairing is associative and graded commutative with sign $(-1)^{(m+n)(p+q)}$ for $r \geqslant 1$,

(v) the pairing on $E^{\infty}$ is compatible with the filtration of Section 3.7.

Proof. It suffices to consider the cases

$$
X=D_{p, q}^{r}, Y=D_{s, t}^{r}, \text { for } r>1 \text {, and } X=S^{t-m, m}, Y=S^{s-n, n} .
$$

By Lemma 4.5, its proof and Proposition 4.2 we have a commutative diagram

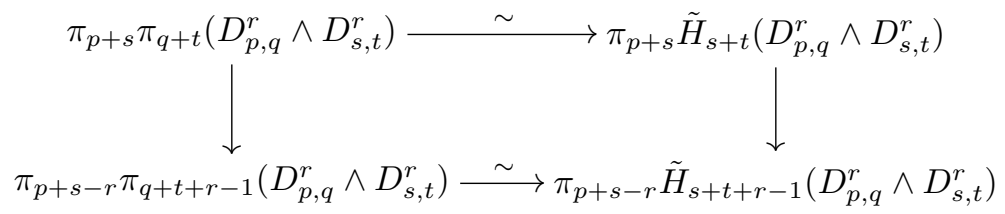

in which the left vertical map is obtained from the homotopy pairing (9) and the right vertical map is obtained from the homology pairing of Theorem 4.1. Properties (i)-(iv) follow readily. The last property follows from Lemmas 4.3 and 4.4.

\section{References}

[1] J.F. Adams, Algebraic Topology - A Student's Guide, Cambridge University Press, London, 1972. London Mathematical Society Lecture Note Series, No. 4.

[2] A.K. Bousfield and E.M. Friedlander, Homotopy theory of $\Gamma$-spaces, spectra and bisimplicial sets, Lecture Notes in Mathematics, 1978, pp. 80-130.

[3] A.K. Bousfield and D.M. Kan, A second quadrant spectral sequence, Trans. AMS 177 (1973), 305-318.

[4] A. Dold and D. Puppe, Homologie nicht-additiver Functoren, Anwendungen, Ann. Inst. Fourier 11 (1961), 201-312.

[5] W.G. Dwyer, D.M. Kan, and C.R. Stover, The bigraded homotopy groups $\pi_{i, j} X$ of a pointed simplicial space X, J. Pure Appl. Algebra 103 (1995), no. 2, 167188.

[6] W.G. Dwyer and J. Spalinski, Homotopy theories and model categories, Handbook of Algebraic Topology, 1995, pp. 73-126.

[7] P.G. Goerss and M.J. Hopkins, Moduli problems for structured ring spectra, 2005. Available from http://www. math.northwestern.edu/ pgoerss.

[8] P.G. Goerss and J.F. Jardine, Simplicial Homotopy Theory, Progress in Mathematics, vol. 174, Birkhäuser Verlag, Basel, 1999.

[9] J.P. May, Simplicial Objects in Algebraic Topology, Chicago Lectures in Mathematics, University of Chicago Press, Chicago, IL, 1992. Reprint of the 1967 original. 
[10] D.G. Quillen, Homotopical Algebra, Lecture Notes in Mathematics, No. 43, Springer-Verlag, New York, 1967.

[11] C.L. Reedy, Homotopy theory of model categories, unpublished 1973 preprint available from http://math. mit.edu/ psh.

[12] C. Rezk, S. Schwede, and B. Shipley, Simplicial structures on model categories and functors, Amer. J. Math. 123 (2001), no. 3, 551-575.

[13] E. Spanier, Algebraic Topology, Springer-Verlag, New York, 1966.

[14] C.A. Weibel, An Introduction to Homological Algebra, Cambridge Studies in Advanced Mathematics, vol. 38, Cambridge University Press, Cambridge, 1994.

[15] G. Whitehead, Elements of Homotopy Theory, Graduate Texts in Mathematics, vol. 61, Springer-Verlag, New York, 1978.

Michael Paluch mike@math.ist.utl.pt

Departamento de Matemática

Instituto Superior Técnico

Technical University of Lisbon

Av. Rovisco Pais

1049-001 Lisbon

Portugal

This article is available at http://intlpress.com/HHA/v9/n2/a1 